4. Almon RR, Dubois DC, Jin JY, Jusko WJ. Temporal profiling of the transcriptional basis for the development of corticosteroid-induced insulin resistance in rat muscle. J Endocrinol. 2005 184(1):219-232

5. Almon RR, DuBois DC, Jusko WJ. A microarray analysis of the temporal response of liver to methylprednisolone: a comparative analysis of two dosing regimens. Endocrinology. 2007;148(5):2209-2225.

6. Saad MJ, Folli F, Kahn JA, Kahn CR. Modulation of insulin receptor, insulin receptor substrate-1, and phosphatidylinositol 3-kinase in liver and muscle of dexamethasone-treated rats. J Clin Invest. 1993;92(4):2065-2072.

7. Buren J, Lai YC, Lundgren M, Eriksson JW, Jensen $\mathrm{J}$. Insulin action and signalling in fat and muscle from dexamethasone-treated rats. Arch Biochem Biophys. 2008;474(1):91-101.

8. Bazuine M, Carlotti F, Tafrechi RSJ, Hoeben RC, Maassen JA. Mitogen-activated protein kinase (MAPK) phosphatase- 1 and -4 attenuate p38 MAPK during dexamethasone-induced insulin resistance in 3T3-L1 adipocytes. Mol Endocrinol. 2004;18(7):1697-1707.

9. Mazziotti G, Gazzaruso C, Giustina A. Diabetes in Cushing syndrome: basic and clinical aspects. Trends Endocrinol Metab. 2011;22(12):499-506.

10. Brennan-Speranza TC, et al. Osteoblasts mediate the adverse effects of glucocorticoids on fuel metabolism. J Clin Invest. 2012;122(11):4172-4189.

11. Foresta $C$, et al. Evidence for osteocalcin production by adipose tissue and its role in human metabolism. J Clin Endocrinol Metab. 2010;95(7):3502-3506

12. Dorheim MA, et al. Osteoblastic gene expression during adipogenesis in hematopoietic supporting murine bone marrow stromal cells. J Cell Physiol. 1993;154(2):317-328.

13. Lee NK, et al. Endocrine regulation of energy metabolism by the skeleton. Cell. 2007;130(3):456-469

14. Fulzele K, et al. Insulin receptor signaling in osteoblasts regulates postnatal bone acquisition and body composition. Cell. 2010;142(2):309-319.

15. Ferron M, Hinoi E, Karsenty G, Ducy P. Osteocalcin differentially regulates beta cell and adipocyte gene expression and affects the development of metabolic diseases in wild-type mice. Proc Natl Acad Sci US A. 2008;105(13):5266-5270

16. Ferron M, McKee MD, Levine RL, Ducy P, Karsenty G. Intermittent injections of osteocalcin improve glucose metabolism and prevent type 2 diabetes in mice. Bone. 2012;50(2):568-575.

17. Heinrichs AA, et al. Identification of multiple glucocorticoid receptor binding sites in the rat osteocalcin gene promoter. Biochemistry. 1993; 32(42):11436-11444.

18. Ducy $\mathrm{P}$, et al. Increased bone formation in osteocalcin-deficient mice. Nature. 1996;382(6590):448-452.

19 . Henneicke $\mathrm{H}$, et al. Corticosterone selectively targets endo-cortical surfaces by an osteoblast-dependent mechanism. Bone. 2011;49(4):733-742.

20. Iglesias P, et al. Serum concentrations of osteocalcin, procollagen type $1 \mathrm{~N}$-terminal propeptide and beta-CrossLaps in obese subjects with varying degrees of glucose tolerance. Clin Endocrinol (Oxf). 2011;75(2):184-188

21. Rosenstock J, et al. The 11-beta-hydroxysteroid dehydrogenase type 1 inhibitor INCB13739 improves hyperglycemia in patients with type 2 diabetes inadequately controlled by metformin monotherapy. Diabetes Care. 2010;33(7):1516-1522.

22. De Bosscher K. Selective clucocorticoid receptor modulators. J Steroid Biochem Mol Biol. 2010; 120(2-3):96-104.

\title{
Amazing IL-9: revealing a new function for an "old" cytokine
}

\section{Edgar Schmitt and Tobias Bopp}

Institute for Immunology, University Medical Centre, Johannes Gutenberg University Mainz, Mainz, Germany.

\begin{abstract}
The incidence of melanoma - the most aggressive form of skin cancer - is dramatically increasing, while the development of innovative therapeutic strategies continues to be challenging, especially due to a lack of knowledge about the molecular mechanisms underlying melanoma progression as well as antitumor immunity. In this issue of the JCI, Yong Lu and colleagues report a central role for Th9 cells in antitumor immunity.
\end{abstract}

Pro-inflammatory cytokines have long been regarded as beneficial in robust antitumor immunity. Specifically, cytokines of the common cytokine receptor $\gamma$ chain family (such as IL-2, IL-15, and IL-21) have been tested for the treatment of melanoma and other tumors in humans (1). However, there is increasing evidence that depending on the individual tumor, inflammation can lead to either favorable or unfavorable clinical prognosis $(2,3)$. Recent clinical trials utilizing monoclonal antibodies blocking the inhibitory molecules programmed cell death 1 (PD-1) (4) or cytotoxic T lymphocyte-associated protein 4 (CTLA-4) (5) suggest that aggravation of an adaptive immune response is a promising therapeutic strategy for the treatment of melanoma.

Conflict of interest: The authors have declared that no conflict of interest exists.

Citation for this article: J Clin Invest. 2012; 122(11):3857-3859. doi:10.1172/JCI65929.
During the last two decades the laboratory of Qing Yi has significantly contributed to our current understanding of potential immunotherapies in human malignancies by describing the role of DCs - and particularly $\mathrm{T}$ cells - in multiple myeloma.

In this issue of JCI, Yong Lu and colleagues from the Yi laboratory elucidate the role of Th9 cells and the $\gamma$ chain family member IL-9 in a B16 melanoma mouse model, and found that Th9-derived IL-9 inhibited tumor progression (ref. 6 and Figure 1). In addition, Th9 cells induce IL-9-dependent expression of CCL20 in lung epithelial cells, thereby promoting the recruitment of $\mathrm{CD} 8 \alpha^{+} \mathrm{CCR} 6^{+} \mathrm{DCs}$, leading to a strong activation of tumorreactive $\mathrm{CCR}^{+} \mathrm{CD}^{+}$CTLs. Collectively, the authors demonstrate an IL-9-based antitumor potency of Th9 cells, which is in quality and quantity superior to the immune response steered by Th1 cells. Hereby, the authors corroborate similar data quite recently published by Purwar et al., showing that IL-9-producing Th9 cells substantially inhibit melanoma growth as well as lung carcinoma growth in a murine B16F10 model (7).

\section{IL-9 restrains tumor progression}

More than two decades ago, IL-9 was identified as p40 or TCGFIII, and it was functionally characterized as a growth factor for repetitively stimulated $\mathrm{T}$ cell lines, but not for naive $T$ cells (8). In addition to its effect on T cells, a mast cell growth-enhancing activity (MEA) was demonstrated, and p40/TCGFIII/MEA was renamed IL-9 (8). Subsequent research proved an essential role for IL-9 in tolerance induction and the pathophysiology of allergic asthma, inflammatory bowl disease, microbial infections, and autoimmunity (8).

In the present study, the authors demonstrate that neutralization of IL-9 in a B16 lung metastasis model resulted in strong tumor growth associated with a significant decrease in the number of $\mathrm{CD} 4^{+} \mathrm{T}$ cells, $\mathrm{CD}^{+} \mathrm{T}$ cells, CD8 $\alpha^{+} \mathrm{DCs}$, and CD $11 \mathrm{~b}^{+}$ DCs in the lung. Further analyses demonstrated that Th9 cell-derived IL-9 prevented tumor progression in prophylactic and therapeutic settings. Previous findings 


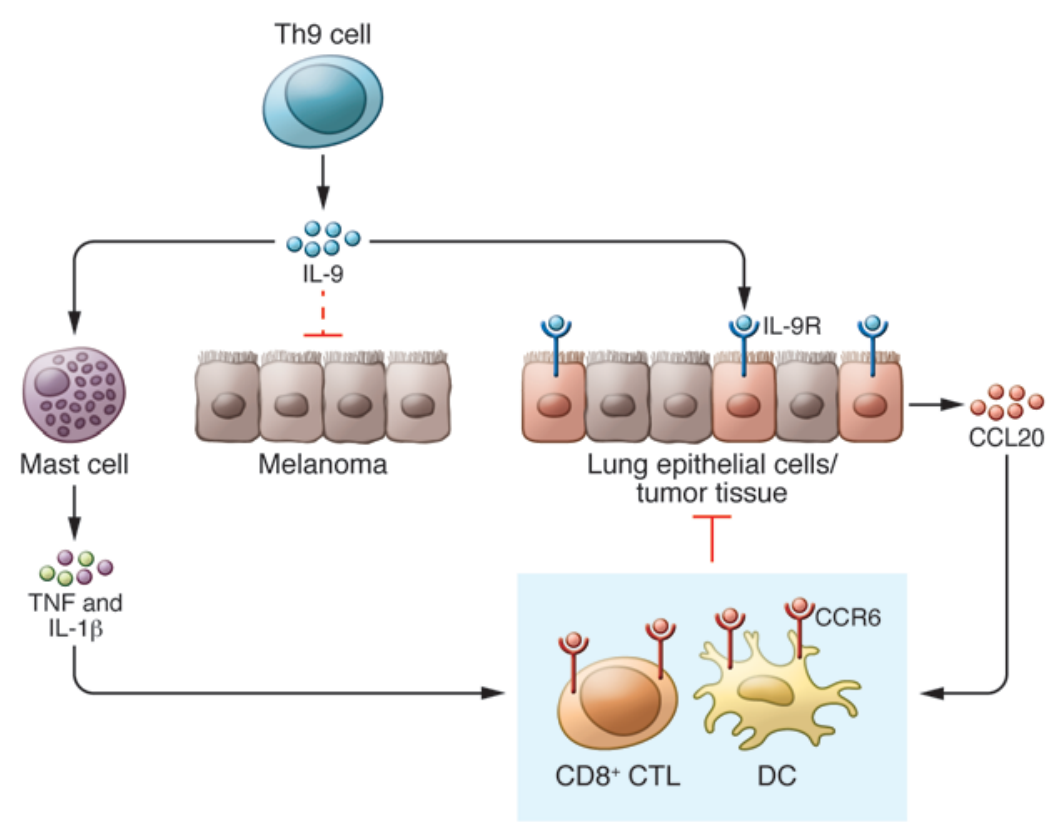

had demonstrated that mutations in the IL9 gene were associated with a higher incidence of cutaneous malignant melanoma (9). However, by its antiapoptotic properties, IL-9 was also characterized as a growth factor for murine $\mathrm{T}$ and $\mathrm{B}$ cell lymphomas in vivo (10), and high levels of IL-9 are associated with myeloid malignancies and Hodgkin disease (11).

Since $\mathrm{Lu}$ et al. reported no direct cytotoxic effect of IL-9 and Th9 cells on B16 melanoma cells, it can be concluded that depending on the expression of the IL-9 receptor, this cytokine can be either beneficial or detrimental for an effective antitumor immune response.

\section{Th9 cell-derived IL-9 induces CCL20} expression in the tumor tissue

IL-9 was shown to induce asthma-like responses in naive mice, as evidenced by increased serum IgE levels, lung eosinophilia, and airway hyperresponsiveness (12). Consequently, neutralization of IL-9 was shown to attenuate this pathology $(13,14)$. Th9 cells seem to contribute to allergic diseases by promoting the expression of the Th2-associated chemokines CCL17 and CCL22 (14). Interestingly, Yong Lu and colleagues now provide evidence that Th9 cells induce the expression of the chemokine CCL20 in lung epithelial cells in an IL-9-dependent manner, thus attracting $\mathrm{CCR}^{+}{ }^{+} \mathrm{DCs}$, which eventually elicit an effective antitumor response. Correspondingly, adoptive transfer of Th9 cells into CCR6-deficient mice failed to improve antitumor immunity. IL-9 was also shown to induce CCL20 expression in astrocytes, suggesting a link between IL-9 and the infiltration of CCR6 $6^{+}$Th17 cells into the central nervous system during neuroinflammation (15). Interestingly, it was recently demonstrated that CCL 20 could be detected in tuberculous pleural effusion as well as in supernatants of cultured pleural mesothelial cells, suggesting that human Th9 cells also express CCR6 (16).

\section{Th9 cells attract CCR6-expressing DCs into tumor tissue, inducing a robust CD8+ CTL-based antitumor immune response}

The role of IL-9 in tumor immunity is far from being fully defined. According to the above cited results, IL-9 obviously plays a rather indirect role, primarily affecting other cell types that ultimately carry out the antitumor immune response. However, it was recently demonstrated that IL-9 promotes the suppressive properties of regulatory $\mathrm{T}$ cells protecting the tumor from being attacked by the immune system (17). In the current manuscript, Lu et al. add to these discrepant results by providing strong evidence that Th9 cells attract CCR6-expressing cells, including tumor-reactive CD8 ${ }^{+}$CTLs and $\mathrm{CD} 8 \alpha^{+} \mathrm{DCs}$, to tumor tissues to induce robust $\mathrm{CD}^{+} \mathrm{CTL}$-driven antitumor immune responses. However, no elevation in mast cell infiltration of the lung was observed. Nevertheless, using mast cell-deficient $\mathrm{Kit}^{\mathrm{W} \text {-sh }}$ sash mice, carrying an inversion mutation

\section{Figure 1}

Effector functions of Th9 cells in antitumor immune responses. Th9-derived IL-9 can have direct antitumor effects or can indirectly influence tumor growth by altering immune responses. IL- 9 induces the production of CCL20 in lung epithelial cells, which mediates the recruitment of CCR6-expressing DCs and CD8 ${ }^{+}$CTLs. In addition, the MEA of IL-9 could further potentiate the induction of an adaptive antitumor immune response by providing mast cell-derived IL-1 $\beta$ and TNF- $\alpha$. IL-9R, IL-9 receptor. upstream of the c-kit gene, Purwar et al. very recently demonstrated that mast cells are required for the antitumor effect of IL-9 (7). This is reminiscent of the MEA that was initially described as a characteristic property of IL-9 (8). This activity could potentially play an important role in the initiation of an antitumor immune response since it was demonstrated that mast cells can initiate immediate early inflammation by enhancing activation and migration of DCs, leading to an accelerated $\mathrm{CD}^{+} \mathrm{CTL}$ response (18).

Hence, the results of Lu et al. and Purwar et al. indicate that Th9-derived IL-9 can play an important protective role in tumor immunology by recruiting other cell types (mast cells, DCs, and CD8 ${ }^{+}$CTLs) that ultimately initiate and maintain an effective antitumor immune response (Figure 1).

\section{Conclusion}

The study by Yong Lu and colleagues provides important novel insights on the promotion of an efficient CD8 ${ }^{+}$CTL-mediated antitumor immune response by $\mathrm{Th} 9$-derived IL-9. In this context, the finding that IL-1 considerably enhances IL-9 production (19) shed new light on historical data from Coley and Fehleisen (reviewed in ref. 20). These two researchers realized that the infection of tumor patients with bacteria occasionally led to a regression of the tumor, and they exploited this observation by generating bacterial-based vaccines (S. pyogenes, S. marcescens) for the treatment of cancer (20). Fever that was caused by these bacterial 
products through the induction of endogenous pyrogenes was thought to play a central role. Later, IL-1 was shown to be a major component of such endogenous pyrogenes, and therefore it can be speculated that the IL-9-potentiating properties of IL-1 represent one of the main antitumor activities of such vaccines. However, IL-9 was also shown to support the growth of tumors that express IL-9 receptors, indicating an ambivalent role of this cytokine in tumor immunology $(10,11)$. Hence, inflammation and in particular distinct pro-inflammatory cytokines may provoke a Janus-faced response depending on the expression of the respective cytokine receptor by tumor cells. This ambivalence implicitly requires a detailed genetic and immunological characterization of each individual tumor and/or patient to develop personalized innovative therapeutic strategies in cancer immunotherapy.

Address correspondence to: Tobias Bopp, Institute for Immunology, Molecular Immunology, University Medical Centre of the Johannes Gutenberg University Mainz,
Mainz, Germany. Phone: 0049.0.6131.176175; Fax: 0049.0.6131.176202; E-mail: boppt@unimainz.de.

1. Dougan M, Dranoff G. Immune therapy for cancer. Annu Rev Immunol. 2009;27:83-117.

2. Leek RD, et al. Association of macrophage infiltration with angiogenesis and prognosis in invasive breast carcinoma. Cancer Res. 1996;56(20):4625-4629.

3. Ribatti D, et al. Tumor vascularity and tryptasepositive mast cells correlate with a poor prognosis in melanoma. Eur J Clin Invest. 2003;33(5):420-425.

4. Topalian SL, et al. Safety, activity, and immune correlates of anti-PD-1 antibody in cancer. $N$ Engl JMed. 2012;366(26):2443-2454.

5 . Hodi FS, et al. Improved survival with ipilimumab in patients with metastatic melanoma. $N$ Engl J Med. 2010;363(8):711-723.

6. Lu Y, et al. Th9 cells promote antitumor immune responses in vivo. J Clin Invest. 2012; 122(11):4160-4171

7. Purwar R, et al. Robust tumor immunity to melanoma mediated by interleukin-9-producing $\mathrm{T}$ cells [published online ahead of print: July 8, 2012]. Nat Med. doi:10.1038/nm.2856.

8. Stassen M, Schmitt E, Bopp T. From interleukin-9 to Thelper 9 cells. Ann N Y Acad Sci. 2012;1247(1):56-68.

9. Yang XR, et al. Identification of modifier genes for cutaneous malignant melanoma in melanomaprone families with and without CDKN2A mutations. Int J Cancer. 2009;125(12):2912-2917.

10. Renauld JC, et al. Thymic lymphomas in interleukin 9 transgenic mice. Oncogene. 1994;9(5):1327-1332.

11. Knoops L, Renauld J-C. IL-9 and its receptor: from signal transduction to tumorigenesis. Growth Factors. 2004;22(4):207-215.

12. Levitt RC, et al. IL-9 pathway in asthma: new therapeutic targets for allergic inflammatory disorders. J Allergy Clin Immunol. 1999;103(5 pt 2):S485-S491.

13. Staudt V, et al. Interferon-regulatory factor 4 is essential for the developmental program of $\mathrm{T}$ helper 9 cells. Immunity. 2010;33(2):192-202.

14. Chang H-C, et al. The transcription factor PU.1 is required for the development of IL-9-producing $\mathrm{T}$ cells and allergic inflammation. Nat Immunol. 2010;11(6):527-534.

15. Zhou Y, et al. IL-9 promotes Th17 cell migration into the central nervous system via CC chemokine ligand-20 produced by astrocytes. J Immunol. 2011;186(7):4415-4421.

16. Ye Z-J, et al. Differentiation and recruitment of Th9 cells stimulated by pleural mesothelial cells in human Mycobacterium tuberculosis infection. PLoS One. 2012;7(2):e31710.

17. Smith SE, Hoelzinger DB, Dominguez AL, Van Snick J, Lustgarten J. Signals through 4-1BB inhibit $\mathrm{T}$ regulatory cells by blocking IL-9 production enhancing antitumor responses. Cancer Immunol Immunother. 2011;60(12):1775-1787.

18. Heib V, et al. Mast cells are crucial for early inflammation, migration of Langerhans cells, and CTL responses following topical application of TLR7 ligand in mice. Blood. 2007;110(3):946-953.

19. Schmitt E, et al. IL-1 serves as a secondary signal for IL-9 expression. J Immunol. 1991;147(11):3848-3854.

20. Nauts HC, Swift WE, Coley BL. The treatment of malignant tumors by bacterial toxins as developed by the late William B. Coley, MD, reviewed in the light of modern research. Cancer Res. 1946;6:205-216.

\title{
Endoplasmic reticulum stress and hypertension - a new paradigm?
}

\author{
Alyssa H. Hasty ${ }^{1}$ and David G. Harrison ${ }^{2}$ \\ 1Department of Molecular Physiology and Biophysics and Division of Clinical Pharmacology, and 2Department of Medicine, \\ Vanderbilt University School of Medicine, Nashville, Tennessee, USA.
}

\begin{abstract}
Hypertension occurs in approximately $30 \%$ of individuals in Western populations and is known to be a major cause of stroke, heart failure, and myocardial infarction. Despite this, the molecular etiology of hypertension remains poorly understood. In this issue of the JCI, Young et al. show that endoplasmic reticulum (ER) stress is an essential signaling event for angiotensin II-induced hypertension in cells of the central nervous system. This provides new insight into the molecular mechanisms that drive hypertension and suggests a potential target for future therapy.
\end{abstract}

In 1940, Irvine Page described a crystalline substance purified from the reaction of renin and renin activator, which he named angiotonin (1). Simultaneously, BraunMenendez and coworkers identified a similar substance, which they called hypertensin

Conflict of interest: The authors have declared that no conflict of interest exists.

Citation for this article: J Clin Invest. 2012; 122(11):3859-3861. doi:10.1172/JCI65173.
(2). It is remarkable that three-quarters of a century later, we are still learning the actions of this molecule that ultimately came to be known as angiotensin II. It is now understood that this octapeptide has pleiotropic actions, including promotion of renal tubular sodium reuptake, aldosterone release, vasoconstriction, vascular remodeling, cardiac hypertrophy, cellular oxidative stress, and inflammation. Ongoing research is constantly refining and expanding this list.
These actions allow survival during stresses such as dehydration or hemorrhage and have pathological roles in numerous diseases including hypertension and heart failure. In addition to its peripheral effects, angiotensin II and its related peptides have potent actions on the central nervous system (3). Certain brain nuclei, predominantly in the hypothalamus and the brain stem, possess all of the components of the renin-angiotensin system (RAS) and produce angiotensin peptides locally (4). These nuclei are physically separated from the peripheral RAS by the blood-brain barrier. In addition, circulating angiotensin II can activate specialized regions of the brain that are adjacent to the cerebral ventricles and lack a well-formed blood-brain barrier. These "circumventricular organs" include the subfornical organ (SFO), the median eminence, the organum 